20xx IEEE. Personal use of this material is permitted. Permission from IEEE must be obtained for all other users, including reprinting/ republishing this material for advertising or promotional purposes, creating new collective works for resale or redistribution to servers or lists, or reuse of any copyrighted components of this work in other works 


\title{
Pilot Based Single User Frequency Offset Estimation in Spectrally-Overlapping FDMA CPM Systems
}

\author{
Nele Noels and Marc Moeneclaey \\ Department for Telecommunications and Information Processing, Ghent University \\ Sint-Pietersnieuwstraat 41, 9000 Gent, Belgium \\ \{nnoels, mm\} @telin. UGent.be
}

\begin{abstract}
The spectral efficiency of a frequency division multiple access system can be increased by allowing some spectral overlap of adjacent user signals, at the expense of higher interuser interference. We derive the linearized mean square error of pilot based single user maximum likelihood frequency offset estimation in such a system, assuming continuous phase modulation. We consider synchronous as well as asynchronous reception of the pilot signals from the various users. Moreover, the pilot signals are assumed to be either constant and equal to 1 , or pseudo-random and independent for all users. In spite of the presence of interuser interference, we obtain relatively simple closed-form expressions, from which the effect of the modulation parameters, the pilot signal structure and the number of users is easily derived.
\end{abstract}

\section{INTRODUCTION}

This paper is concerned with frequency offset (FO) estimation in the reverse link of a multiuser (MU) satellite communication system with timeasynchronous spectrally-overlapping frequency division multiple access (SO-FDMA). Multiple users, at various geographic locations, simultaneously transmit via satellite their single-carrier modulated signal to a receiving basestation, by sharing a common additive white Gaussian noise (AWGN) bandpass channel. The SO-FDMA protocol assigns carrier frequencies to distinct users. In order to increase spectral efficiency, the spacing between the carrier frequencies is made smaller than twice the single-sided bandwidth of the individual user signals, causing each user signal being affected by leakage from neighboring user signals. This leakage signal is referred to as inter-user interference (IUI).

Optimal detection in the case of SO-FDMA signaling is prohibitively complex. Practical receivers comprise a synchronization parameter estimation unit followed by an approximate maximum a posteriori probability (MAP) bit detector. Approximate MAP detectors with a reasonable complexity that yield a very good performance have recently been proposed for continuous phase modulations (CPM) [1]-[5]. CPM is a modulation method commonly used in wireless modems [6]. The transmitted CPM waveform has a constant envelope, and its phase is a continuous function of time that changes according to the digital information to be transmitted. CPM is attractive because of its high efficiency in terms of both power and bandwidth, and because of its robustness to nonlinearities. Proper operation of the detectors from [1]-[5] and references therein requires that accurate estimates of the FOs and time delays associated with the different users are available at the receiver. Acquiring these estimates from the noisy observation of the received MU signal is a difficult task that has received only little attention in the open literature. The current paper focuses on FO estimation, assuming that the timing of the different users is perfectly known at the receiver. In a practical SO-FDMA system, the time delays are not a priori known and will have to be estimated prior to FO recovery.

It is common practice that the users, besides the data modulated signal, also transmit an a priori known pilot signal, to aid time delay and FO estimation at the receiving basestation. The pilot signals are active during dedicated time intervals that comprise only a small fraction of the total transmission time. The asynchronous MU signaling causes the pilot signal intervals of the different users to be asynchronously observed at the receiver. This substantially complicates the joint pilot-based (PB) estimation of all the FOs from the various users, so that suboptimal $\mathrm{PB}$ single user (SU) estimation methods, operating in the presence of IUI, have to be used instead. SU PB FO estimation is performed independently for each user. In the following we derive closed-form expressions for the linearized mean squared estimation error 
(MSEE) of the PB SU maximum likelihood (ML) FO estimator in the presence of IUI due to SOFDMA. These expressions will allow us to quantify the effect of the modulation parameters, the pilot signal structure and the number of users.

\section{System Model AND FO Estimation}

$U$ users are simultaneously active in partially overlapping subbands of a common frequency band with bandwidth $B_{t o t}$, around a given center frequency $\nu_{c}$. They transmit frames of duration $D T$, that are separated by idle periods of duration $N_{\max } T$. For $t \in[0, D T]$, the user with index $u \in\{1,2, \ldots, U\}$ transmits a continuous-phase, constant-envelope, unitpower signal $s_{B B}(t ; u)$. The frequency content of the signals $s_{B B}(t ; u)$ is mainly concentrated in a band $\left[-\frac{B}{2}, \frac{B}{2}\right]$, with $B$ considerably smaller than $B_{t o t}$ but slightly larger than $B_{t o t} / U$. For $t \in[0, N T]$, the signal $s_{B B}(t ; u)$ equals the pilot signal $s_{B B, p}(t ; u)$ of duration $N T$, which is a priori known and transmitted to ease synchronization parameter estimation at the receiver. For $t \in[N T, D T], s_{B B}(t ; u)$ equals the CPM data signal $s_{B B, d}(t ; u)$ of duration $(D-N) T$, which serves to transmit $D-N$ unknown data symbols $a_{n}^{(u)}, n=N, N+1, \ldots, D-1$. The symbols $\left\{a_{n}^{(u)}\right\}$ are assumed to be independent and uniformly distributed (UD) over $\{ \pm 1, \pm 3, \ldots, \pm(M-1)\}$. The relationship between the CPM data signal $s_{B B, d}(t ; u)$ for $t \in[N T, D T]$ and the data symbols $a_{n}^{(u)}, n=$ $N, N+1, \ldots, D-1$ is the following:

$s_{B B, d}(t ; u)=s_{B B, p}(N T ; u) e^{j 2 \pi \frac{K}{P} \sum_{n=N}^{D-1} a_{n}^{(u)} q(t-n T)}$

In (1), $K / P$ is the modulation index ( $K$ and $P$ are relatively prime integers). The function $q(t)$ is the phase-smoothing response, which is related to the frequency pulse $\dot{q}(t)$ by the relationship $q(t)=$ $\int_{0}^{t} \dot{q}(u) d u$.

Equally spaced carrier frequencies are assigned to the different users. The normalized (to the symbol interval $T$ ) frequency shift $f^{(u)}$ of the $u$ th user with respect to $\nu_{c}$ is given by: $f^{(u)}=(u-0.5(U+1))$. $\Delta_{f}$, with $\Delta_{f}$ denoting the normalized carrier spacing; this spacing is slightly smaller than $B T$ and such that $(U-1) \Delta_{f}+B T$ does not exceed $B_{t o t} T$.

At the receiving basestation, the observed bandpass signal, which contains the contributions from all $U$ users, is down-converted to the frequency band $\left[-\frac{B_{t o t}}{2}, \frac{B_{t o t}}{2}\right]$ and low-pass filtered. Assuming ideal power control such that all signals are received with the same power, we normalize the received signal such that each user signal has unit power; the resulting complex baseband received signal after normalization can be modeled as:

$$
r(t)=\sum_{u=1}^{U} s^{(u)}(t)+n(t),
$$

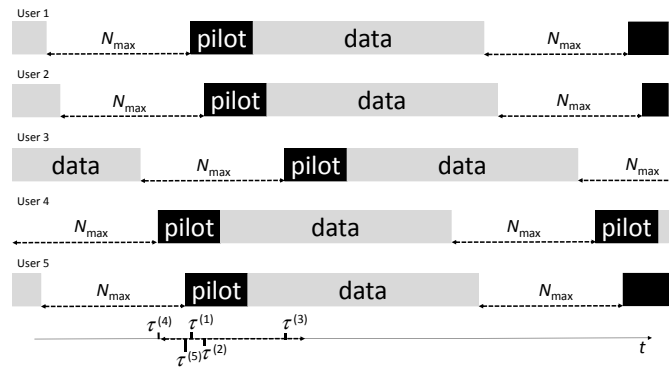

Figure 1. Asynchronous reception of the signals from the different users in a 5 user SO-FDMA system.

where $n(t)$ is zero mean (ZM) circular symmetric $(\mathrm{CS})$ complex-valued $(\mathrm{CV}) \mathrm{AWGN}$ with power spectral density $T N_{0} / E_{s}$, with $N_{0}$ the noise power spectral density and $E_{s}$ the energy per symbol period before normalization, and $s^{(u)}(t)$ is the unit-power contribution from user $u$ to the normalized received signal:

$$
s^{(u)}(t)=s_{B B}^{(u)}(t) e^{j 2 \pi\left(f^{(u)}+F^{(u)}\right) \frac{t-\tau^{(u)}}{T}} e^{j \theta^{(u)}},
$$

with $s_{B B}^{(u)}(t)=s_{B B}\left(t-\tau^{(u)} ; u\right)$, where $\tau^{(u)}$ is the time delay associated with the $u$ th user; these delays incorporate the propagation delays and some asynchronicity of the user's frame tranmission instants. The quantities $\theta^{(u)}$ and $F^{(u)}$ stand for the phase shift and the normalized FO of the signal from user $u$. $F^{(u)}$ is assumed to be much smaller than both $\Delta_{f}$ and $1 / T$. The phase shifts $\left\{\theta^{(u)}\right\}$ and the time delays $\left\{\tau^{(u)}\right\}$ are modelled as independent random variables (RV) with $\tau^{(u)}$ UD over $\left[0, N_{\max } T\right]$ and $\theta^{(u)}$ UD over $[-\pi, \pi]$. In the remainder of this paper it is further assumed that $N_{\max }$ is smaller than $(D-N)$. Because of the idle periods of duration $N_{\max } T$ between frames, a frame received from the $u$ th user is interfered by exactly one frame from each of the other $U-1$ users, as illustrated in Fig. 1. State-of-the-art SO-FDMA detectors jointly decode the information from the different users by applying an iterative soft-interference cancellation algorithm.

The PB SU ML FO estimate $\hat{F}^{(u)}$ of $F^{(u)}$ is the value of $\tilde{F}$ that maximizes the probability density function of the signal $r(t)$, conditioned on $F^{(u)}=\tilde{F}$, with the receiver assuming that only the pilot symbols from the $u$ th user were transmitted (i.e., the receiver ignores the data part of the frame from the $u$ th user and the frames from the $U-1$ interfering users). We further assume that the estimator knows the time delay $\tau^{(u)}$ (e.g., via a preceding timing synchronization unit). Taking into account that $\left|F^{(u)}\right| \ll 1$, the ML estimation reduces to

$$
\hat{F}^{(u)}=\arg \max _{\tilde{F}} X^{(u)}(\tilde{F}),
$$


with $X^{(u)}(\tilde{F})=\left|Y^{(u)}(\tilde{F})\right|^{2}$, where $Y^{(u)}(\tilde{F})$ is given by:

$$
Y^{(u)}(\tilde{F})=\sum_{n=0}^{N-1} e^{-j 2 \pi \tilde{F} n} z_{n}^{(u)}
$$

and $z_{n}^{(u)}$ is defined as:

$z_{n}^{(u)}=\frac{1}{T} \int_{n T}^{(n+1) T} r\left(t+\tau^{(u)}\right) e^{-j 2 \pi f^{(u)} \frac{t}{T}} s_{B B, p}^{*}(t ; u) d t$.

The accuracy of $\hat{F}^{(u)}$ is generally assessed in terms of the MSEE $\sigma^{2}(u ; U)=\mathbb{E}\left[\left(\hat{F}^{(u)}-F^{(u)}\right)^{2}\right]$, with $\mathbb{E}[$.$] denoting the statistical average with respect to$ $r(t)$, given $F^{(u)}$. An exact closed-form expression $\sigma^{2}(u ; U)$ is difficult to obtain. For small FO estimation errors, the following linearization applies [7]:

$$
\sigma^{2}(u ; U) \approx \mu_{2, \dot{X}}^{(u)} /\left(\mu_{\ddot{X}}^{(u)}\right)^{2},
$$

where $\mu_{2, \dot{X}}^{(u)}$ and $\mu_{\ddot{X}}^{(u)}$ denote $\mathbb{E}\left[\left(\dot{X}^{(u)}\right)^{2}\right]$ and $\mathbb{E}\left[\ddot{X}^{(u)}\right]$, respectively. Here and further throughout this paper we use short-hand notations $Q^{(u)}, \dot{Q}^{(u)}$ and $\ddot{Q}^{(u)}$ to represent the zeroth, first and second order derivative of a function $Q^{(u)}(F)$ of $u$ and $F$ with respect to $F$, and evaluated in $F=F^{(u)}$.

The linearized MSEE (7) depends on the properties of the pilot signals and the inter-user time delay differences $\Delta \tau^{(v, u)}=\tau^{(v)}-\tau^{(u)}$. For the pilot signals themselves, two cases are considered. In the first case, denoted (ra), the pilot signals result from applying CPM to a random sequence of $N$ statistically independent (SI) pilot symbols that are UD over $\{ \pm 1, \pm 3, \ldots, \pm(M-1)\}$ and independent among users. In the second case, denoted (co), all users have the same pilot signal, which equals 1 over the interval $[0, N T]$. Regarding the time delay differences, we consider two extreme cases. In the first case, denoted (sync), we assume that the frames for the different users arrive synchronously at the receiver, so that the pilot signals from all users overlap during a time period equal to $N T$; this is a simplified model for the situation where the time delay uncertainty $N_{\max } T$ is much smaller than the pilot signal duration $N T$. In contrast, the second case, denoted (async), considers a large amount of asynchronism. In this case, $N_{\max }$ is assumed large as compared to $N$ (but smaller than $D$ ), such that the pilot signal of any user almost surely overlaps with either the data signals (with probability close to 0.5) or the idle time of the other users. Combining the two cases of the delay difference with the two cases of the pilot signals gives rise to four scenarios (sync, co), (sync, ra), (async, co), (async, ra), which are investigated below.

\section{Derivation of Linearized MSEE}

A. Decomposition of $Y^{(u)}(\tilde{F})$

By substituting (2) into (6), the function $Y^{(u)}(\tilde{F})$ from (5) decomposes as follows

$$
\begin{aligned}
& Y^{(u)}(\tilde{F}) \\
& =Y_{\text {sig }}^{(u)}(\tilde{F})+\sum_{v \neq u} Y_{I U I}^{(v, u)}(\tilde{F})+Y_{A W G N}^{(u)}(\tilde{F}) .
\end{aligned}
$$

Here,

$$
\begin{gathered}
Y_{\text {sig }}^{(u)}(\tilde{F})=e^{j \theta^{(u)}} \sum_{n=0}^{N-1} e^{j 2 \pi\left(F^{(u)}-\tilde{F}\right) n}, \\
Y_{I U I}^{(v, u)}(\tilde{F})=e^{j \theta^{(v)}} \sum_{n=0}^{N-1} e^{j 2 \pi\left(F^{(v)}-\tilde{F}\right) n} \zeta_{I U I, n}^{(v, u)},
\end{gathered}
$$

and

$$
Y_{A W G N}^{(u)}(\tilde{F})=\sum_{n=0}^{N-1} e^{-j 2 \pi \tilde{F} n} W_{n}
$$

The quantity $\zeta_{I U I, n}^{(v, u)}$ in (10) is defined as

$$
\zeta_{I U I, n}^{(v, u)}=\frac{1}{T} \int_{0}^{T} h_{n}^{(u, v)}(t) e^{j 2 \pi \Delta_{f}(v-u) \frac{t+n T}{T}} d t,
$$

with

$h_{n}^{(v, u)}(t-n T)=s_{B B}\left(t-\Delta \tau^{(v, u)} ; v\right) s_{B B, p}^{*}(t ; u)$

and $\Delta \tau^{(v, u)}=\tau^{(v)}-\tau^{(u)}$. The quantities $W_{n}, n=$ $0,1, \ldots, N-1$ in (11) are SI ZM CS CV Gaussian RVs with variance $N_{0} / E_{s}$.

\section{B. Derivation of $\sigma^{2}(u ; U)$}

Taking into account that $Y_{A W G N}^{(u)}(\tilde{F}), Y_{\text {sig }}^{(u)}(\tilde{F})$ and $Y_{I U I}^{(v, u)}(\tilde{F})$ are ZM SI RVs, we can write:

$$
\begin{aligned}
& \mathbb{E}\left[X^{(u)}(\tilde{F})\right] \\
& \approx\left|Y_{\text {sig }}^{(u)}(\tilde{F})\right|^{2}+\mathbb{E}\left[\left|Y_{A W G N}^{(u)}(\tilde{F})\right|^{2}\right], \\
& =\left(\sum_{n, m=0}^{N-1} e^{-j 2 \pi\left(\tilde{F}-F^{(u)}\right)(n-m)}+\frac{E_{s}}{N_{0}} N\right),
\end{aligned}
$$

where the approximation holds for $\tilde{F}$ in the vicinity of $F^{(u)}$ and for small IUI. Taking the second derivative in $\tilde{F}=F^{(u)}$ yields, for large $N$

$$
\mu_{\ddot{X}}^{(u)}=-\frac{2}{3} \pi^{2} N^{4} .
$$

To compute $\mu_{2, \dot{X}}^{(u)}$ we express $\left(\dot{X}^{(u)}\right)^{2}$ as function of $Y_{\text {sig }}^{(u)}, \dot{Y}_{s i g}^{(u)}, Y_{A W G N}^{(u)}, \dot{Y}_{A W G N}^{(u)}, Y_{I U I}^{(v, u)}$ and $\dot{Y}_{I U I}^{(v, u)}$, and exploit their statistical properties. Assuming weak IUI, we find

$$
\mu_{2, \dot{X}}^{(u)}=\mu_{2, \dot{X}, A W G N}^{(u)}+\sum_{v \neq u} \mu_{2, \dot{X}, I U I}^{(v, u)},
$$


where $\mu_{2, \dot{X}, A W G N}^{(u)}$ and $\mu_{2, \dot{X}, I U I}^{(v, u)}$ denote the contributions caused by the AWGN and by the IUI from user $v \neq u$, respectively. From the case where $U=1$, it is known that, for large $N$ [7], the first term in (15) is given by:

$$
\mu_{2, \dot{X}, A W G N}^{(u)}=\frac{2}{3} \pi^{2} N^{5} \frac{N_{0}}{E_{s}} .
$$

The computation of the remaining terms in (15) is straightforward but tedious. The result is that for large $N$, the linearized MSEE is given by

$$
\sigma^{2}(u ; U)=\frac{3}{2 \pi^{2} N^{3}}\left(\frac{N_{0}}{E_{s}}+\sum_{v \neq u} \mathcal{V}\left(\Delta_{f}|v-u|\right)\right),
$$

where

$$
\mathcal{V}(f)= \begin{cases}\frac{6}{N} \frac{\cos ^{2}(\pi f N)}{(\pi f)^{2}} & , \text { (sync, co) } \\ \frac{1}{T} \mathcal{F}\left\{\left(\langle\mathcal{C}(\tau, \tau+t)\rangle_{\tau}\right)^{2}\right\}_{f} & , \text { (sync, ra) } \\ \frac{1}{2 T} \mathcal{F}\left\{\langle\mathcal{C}(\tau, \tau+t)\rangle_{\tau}\right\}_{f} & , \text { (async, co) } \\ \frac{1}{2 T} \mathcal{F}\left\{\left(\langle\mathcal{C}(\tau, \tau+t)\rangle_{\tau}\right)^{2}\right\}_{f} & , \text { (async, ra) }\end{cases}
$$

Due to space constraints, we omit the proof. In (18), $\mathcal{C}\left(t_{1}, t_{2}\right)=\mathbb{E}\left[\tilde{s}\left(t_{1}\right) \tilde{s}^{*}\left(t_{2}\right)\right]$ is the real-valued autocorrelation function $\mathcal{C}\left(t_{1}, t_{2}\right)=\mathbb{E}\left[\tilde{s}\left(t_{1}\right) \tilde{s}^{*}\left(t_{2}\right)\right]$ of the cyclostationary (with respect to the time variable) random process $\tilde{s}(t)$ that results from extending the support of $s_{B B, d}(t ; u)$ to $(-\infty, \infty)$, $\langle C(\tau, \tau+t)\rangle_{\tau}$ is short-hand for $\frac{1}{T} \int_{0}^{T} C(\tau, \tau+t) d \tau$ and $\mathcal{F}\{x(t)\}_{f}=\int_{-\infty}^{\infty} x(t) e^{-j 2 \pi f t} d t$ denotes the Fourier transform of $x(t)$.

The linearized MSEE from (17) decomposes into an AWGN contribution and an IUI contribution. The AWGN contribution is the same for all scenarios, inversely proportional to the signal-to-noise ratio (SNR) $E_{s} / N_{0}$ and also inversely proportional to $N^{3}$. The IUI contribution is independent of the SNR. It depends on the number of users $U$ in the system, on the considered scenario and on the selected SO-FDMA CPM format. This contribution is inversely proportional to $N^{4}$ for (sync, co) and inversely proportional to $N^{3}$ for (sync, ra), (async, co) and (async, ra). It is two times larger for (sync, ra) than for (async, ra), a direct result of the users' idle times (see Fig. 1).

The generalization of (17)-(18) for an arbitrary $N_{\text {max }}$, in which case a segment of the pilot signal and/or a segment of the data signal from a same interfering user can affect the pilot signal of the considered user (e.g., user 4 interfering with user 2 in Fig. 1), is work in progress.

\section{NUMERICAL RESULTS AND DISCUSSION}

Numerical results are presented for a SO-FDMA CPM scheme from [1]-[5] with $\dot{q}(t)=\frac{1}{2 T}, t \in$ $[0,2 T], \frac{K}{P}=\frac{1}{3}, M=2$ and $\Delta_{f}=0.3$. Note that, for the particular CPM scheme considered, pilot signals

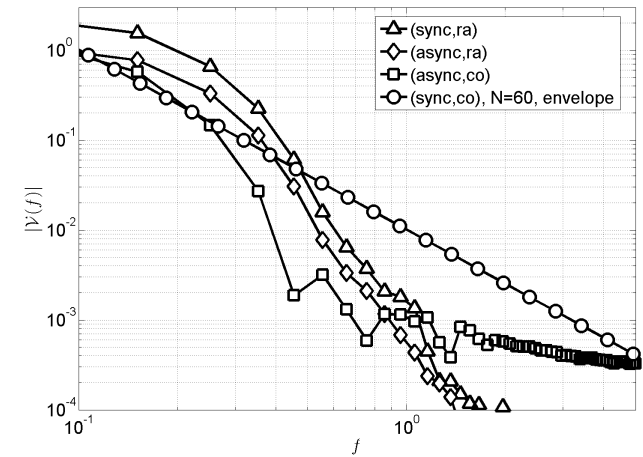

Figure 2. $\mathcal{V}(f)$ from (18) for $\dot{q}(t)=\frac{1}{2 T}, t \in[0,2 T], \frac{K}{P}=\frac{1}{3}$ and $M=2$.

that are constant and equal to 1 result from choosing pilot symbol sequences that alternate between of -1 and +1 . Depending on the channel coding, the number of users $U$ and the detection algorithm, the operating SNR values reported for this scheme are in the range $[6 \mathrm{~dB}, 9 \mathrm{~dB}]$.

Fig. 2 shows the corresponding $\mathcal{V}(f)$ from (18) for (sync, ra), (async, co) and (async, ra) together with the envelope $\mathcal{V}_{e}(f)=\frac{6}{N \pi^{2} f^{2}}$ of $\mathcal{V}(f)$ for (sync, co) and $N=60$. For all scenarios, $\mathcal{V}(f)$ goes to zero for large $f$. This implies that, for large $U$, the IUI contribution to (17) becomes independent of $U$. The value $U_{\text {eff }}$ of $U$ at which this occurs decreases with the spectral spacing between the users, and depends on the modulation format (for (sync, ra), (async, co) and (async, ra)) or on the pilot signal duration $N$ (for (sync, co)).

The obtained linearized MSEE expressions are now numerically evaluated for $u=u_{c}=\frac{U+1}{2}$, $N \in\{60,120\}$ and $U \in\{5,17\}$, and compared to the result for $U=1$. The number of data symbols $(D-N)$ was set to 1297 . The actual FOs were modeled as

$$
F^{(u)}=F_{A}+F_{B}^{(u)},
$$

with $F_{A}$ UD in $[-0.005,0.005]$ (common to all users) and $F_{B}^{(u)}$ UD in $[-0.001,0.001]$ (SI among users). The search in (4) is performed over all $\tilde{F}$ in $[-0.006,0.006]$, which is the actual range of $F^{(u)}$. Fig. 3 shows the ratio $\sigma^{2}\left(u_{c} ; U\right) / N^{3}$ as a function of the SNR.

- At low SNR, the AWGN dominates the estimation performance so that all curves converge to the MSEE for $U=1$.

- Due to the IUI contribution a MSEE floor occurs at high $E_{s} / N_{0}$. At SNRs above a given threshold, the estimator operates in a merely IUIlimited, rather than a noise-limited regime.

- The linear approximation of $\sigma^{2}\left(u_{c} ; U\right) / N^{3}$ for (async, co), (async, ra) and (sync, ra) is in- 


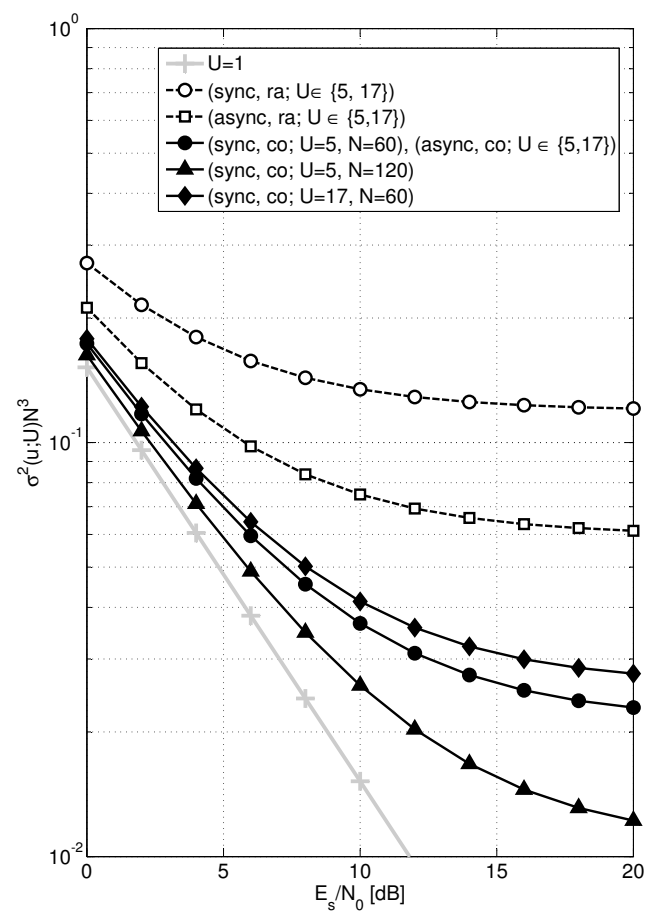

Figure 3. Theoretical and simulated normalized MSEE for SOFDMA CPM scheme with $\dot{q}(t)=\frac{1}{2 T}, t \in[0,2 T], \frac{K}{P}=\frac{1}{3}$, $M=2$ and $\Delta_{f}=0.3$.

dependent of $N$ (see (17)-(18)). Fig. 3 further shows that the corresponding MSEEs do not visibly change when going from $U=5$ to $U=17$. This is in accordance with (17) and the observation from Fig. 2 that, for these scenarios, $\mathcal{V}(f)$ virtually vanishes for $f$ larger than 1 , which is about 3 times $\Delta_{f}$.

- At high SNR, the linearized MSEE for (sync, ra) is twice as large as for (async, ra) (see (2)), which in turn is significantly larger than for (async, co) (see Fig. 3). The latter observation is in accordance with (18) and with the observation from Fig. 2 that $\mathcal{V}\left(\Delta_{f}\right)$ and $\mathcal{V}\left(2 \Delta_{f}\right)$ which determine the IUI contribution to (17) from the nearest and second nearest neigbours are larger for (async, ra) than for (async, co).

- For (sync, co), the linearized MSEE at high
SNR strongly decreases (increases) when $N$ $(U)$ increases. This is in accordance with (17) and with the observation that $\mathcal{V}(f)$ decreases considerably slower with $f$ for (sync, co) than for the other scenarios. Hence, $U_{\text {eff }}$ is significantly larger for (sync, co) than for (sync, ra), (async, co) and (async, ra).

- In general, the SU PB ML FO estimation performance is significantly better for co than for ra.

\section{CONCLusions}

We have derived closed-form analytical expressions for the linearized MSEE of SU PB ML FO estimation in synchronous and severely asynchronous SOFDMA CPM communication systems. These expression provide valuable insights with respect to the effect on the MSEE of the pilot signal duration, the number of interfering users, the type of pilot signal and the level of asynchronism. We have validated our analytical results comparing their numerical evaluation with simulation results.

\section{ACKNOWLEDGMENT}

This research has been funded by the Interuniversity Attraction Poles Program initiated by the Belgian Science Policy Office. The first author would like to acknowledge the financial support of the Fund for Scientific Research - Flanders (FWO Vlaanderen).

\section{REFERENCES}

[1] N. Noels and M. Moeneclaey. Iterative multiuser detection of spectrally efficient FDMA CPM. IEEE Trans. Signal Processing, 60:5254-5267, October 2012.

[2] A. Perotti et al. Spectrally efficient multiuser continuous-phase modulation systems. In IEEE Int. Conf. Commun. (ICC), Cape Town, South Africa, May 2010.

[3] A. Piemontese et al. Information-theoretic analysis and practical coding schemes for spectrally efficient fdm-cpm systems. In 6th Int. Symposium on Turbo Codes and Iterative Inf. Processing (ISTC), Brest, France, September 2010.

[4] A. Piemontese et al. Improving the spectral efficiency of FDM$\mathrm{CPM}$ systems through packing and multiuser processing. In 11th signal processing for space commun. workshop (SPSC), Cagliari, Italy, September 2010.

[5] A. Piemontese and G. Colavolpe. A novel graph-based suboptimal multiuser detector for fdm-cpm transmissions. IEEE Trans. Wireless Commun., 9:2812-2819, September 2010.

[6] T. Aulin et al. Continuous phase modulation. IEEE Trans. Commun., 29:196-225, March 1981.

[7] H. Meyr et al. Digital Communication Receivers. John Wiley \& Sons Inc., Hoboken, NY, 1st edition, 1998. 